The case of blue sclerotic with blue discolouration of the face mentioned in the beginning of the article was in no way connected with the hereditary group already described. It was noted in a Multani girl of a blonde type, aged 9 years, the sclerotic of whose right eye was of deep blue colour and who had an adherent leucoma of the same eye. The right half of the face had a faint blue discolouration. The discoloured area extended a little beyond the middle line, nearly up to it on the lower jaw and as far as the right ear. The right side of the nose and upper lid had escaped the discolouration. At the periphery the blue colour gradually faded away into the normal tint of the skin. The skin in the affected area was not thickened and did not show any dilated blood-vessels. The eye except for the adherent leucoma and blue sclerotic was of normal size and shape. No other member of the family was similarly affected though she herself was born with it. Before a photograph of the face could be arranged for she disappeared.

\title{
Remarks
}

(1) All cases of the hereditary type were bilateral.

(2) No evidence of fragilitas ossium, deafness, or congenital syphilis could be obtained.

(3) All cases were free from error of refraction except one that was myopic.

(4) All the affected members of the family were females with only one exception, that being a slightly affected male.

(5) The affection was transmitted through females to the descendants and was dying out through the only male member of the family.

\section{A FAMILY WITH ANIRIDIA}

$\mathrm{BY}$

Major J. N. DugGan, D.(O.(Oxon), F.C.P.S., L.M. \& S.(Bom.), AND

B. P. Nanavati, D.O.M.S., M.R.C.S.(Eng.)

IT is not often that one comes across cases of aniridia, much less so as a familial disease, and hence the following cases are considered worthy of record :

Family History.-All the affected children were the offspring of the third wife of their father, those by the first two being quite 
normal. There was no history of any eye trouble on the father's side. The mother who died when she was aged 44 years, had nystagmus and poor sight. Their maternal aunt, who is still alive; was said to have gone blind a couple of years ago, but no evidence of nystagmus could be gathered from the lady who brought the children to the Sir C. J. Eye Hospital, Bombay. There was the history of a single miscarriage.

The family consisted of one girl and three boys. All of them had aniridia and varying degrees of nystagmus with myopia.

Case No. 1.-Miss H.K., aged 9 years, whose eye looked rather prominent, had bilateral hereditary horizontal nystagmus of moderate rapidity of movement. Cornea was normal. Only a narrow band of iris about $1 \mathrm{~mm}$. wide, could be made out in each eye. Visual acuity of each eye was found to be $6 / 24$, improved to 6/18 partly with $-2 \mathrm{D}$. sph. and $-3 \mathrm{D}$. cyl. axis horizontal. Ophthalmoscopically a very narrow rim of iris was seen, though in the left eye between five and seven o'clock it was wider than elsewhere. Lens of each eye was clear and its border round and regular. Efforts were made to see the fibres of the suspensory ligament and ciliary processes in the circumlental space which could be easily widened by making the patient look down, but neither could be made out with the ophthalmoscope. There were no vitreous opacities. Each fundus was normal and did not show any pigmentary disturbance anywhere.

Case No. 2.-Master P.K., aged 14 years, presented the appearance of aniridia in each eye, though the rim of iris, especially that of the left eye, was wider than that of case No. 1. Though no nystagmus was present, by making the patient look in the extreme horizontal positions, several nystagmoid jerks could be obtained in each position. Tension was normal. Visuall acuity was $6 / 6$ with $-6 \mathrm{D}$. sph. With the ophthalmoscope, a narrow rim of iris, which was wider in the left eye between five and seven o'clock than in the other part of the circumference, was seen. Each lens was clear and its border was regular and round. Fine vitreous opacities were noted. No suspensory ligament or ciliary processes could be made out. Both fundi were normal and there was no pigmentary disturbance.

Case No. 3.-Master A.K., aged 17 years, had bilateral horizontal hereditary type of nystagmus. Right eye, the cornea was of normal size. No rim of iris was seen in this case. The diffusely opaque lens with a regular border was subluxated upwards and inwards, but was not tremulous. A black crescentic area below the lower border of the subluxated lens attracted attention. Left eye, the cornea was faintly opaque and was larger than the right. The sclerotic was blue and the eye which as a whole looked 
enlarged was congested and resembled a case of buphthalmos: The opaque lens could be seen moving about behind the cornea and seemed attached to the eyeball on the upper and inner side. There was xerosis of conjunctiva of both eyes. Tension of the left eye was much higher than that of the right.

His right eye previously had good vision, but for the last year and a half it had been failing. No reliable history of the vision or of any inflammation of the left eye could be obtained. On examination, the right eye was found to recognize hand movements and the left eye was totally blind. Formerly he was using $-6 \mathrm{D}$. sph. before the right eye.

With the ophthalmoscope, in the case of the right eye, a faint fundal glow could be obtained through the crescentic area below in which no rim of iris was seen. With the eye looking down, a white patch of fairly large size and irregular appearance with several white dots without any pigment scattered round it could be seen in the periphery of the fundus (probably choroidal atrophy). Projection was absent above. In the left eye there was no perception of light.

Case No. 4.-Master K.K., aged 11 years, had interstitial keratitis and aniridia in both eyes. He had a very rapid bilateral nystagmus of horizontal type. The vision of the right eye was fingers at four feet, not improved with glasses, and that of the left eye fingers at ten feet, with - 11D. sph., 6/60. Ophthalmoscopically the left eye showed a narrow rim of iris. Fundus and media were normal. There was no pigmentary disturbance in any part of the fundus.

Wassermann was negative in all cases.

Teeth in all cases were found to be carious and misplaced but not notched. There were no signs of congenital syphilis.

\section{Summary}

(1) All the cases of aniridia seemed to have inherited the defect from their mother who had nystagmus, bad sight, and probably aniridia.

(2) All the cases had myopia and nystagmus except one in whom nystagmoid jerks could be obtained.

(3) In none could suspensory ligament, ciliary processes, or pigmentary disturbance in the fundus be seen.

(4) In one case interstitial keratitis was present. In another boy subluxated opaque lens in each eye and buphthalmos on one side were found. In the last case, a white patch probably of choroidal atrophy was noted. 